\title{
Clinical Value of the Postpacing Interval for Mapping of Ventricular Tachycardia in Patients with Prior Myocardial Infarction
}

\author{
FRANK BOGUN, M.D., * BRADLEY KNIGHT, M.D., RAJIVA GOYAL, M.D., \\ S. ADAM STRICKBERGER, M.D., STEFAN H. HOHNLOSER, M.D.,** \\ and FRED MORADY, M.D.
}

From the Division of Cardiology, Department of Internal Medicine, University of Michigan Medical Center, Ann Arbor, Michigan; and the *Division of Cardiology, J.W. Goethe University, Frankfurt, Germany

\begin{abstract}
Postpacing Interval. Introduction: The postpacing interval (PPI) has been used to discriminate bystander sites from critical sites within a ventricular tachycardia (VT) reentry circuit, with a PPI that is similar to the VT cycle length (CL) being indicative of a site within the reentry circuit. The purpose of this study was to assess the clinical value of the PPI for identifying effective target sites for ablation of VT at sites of concealed entrainment in patients with prior myocardial infarction.

Methods and Results: In 24 patients with coronary artery disease and a past history of myocardial infarction, 36 VTs with a mean CL of $483 \pm 80 \mathrm{msec}( \pm$ SD) were mapped and targeted for radiofrequency (RF) ablation. The only criterion used to select target sites for ablation was concealed entrainment. In a post hoc analysis, the PPI was measured at 47 ineffective and 26 effective ablation sites. The mean PPI-VTCL difference at the 26 effective sites $(114 \pm 137 \mathrm{msec})$ did not differ significantly from the mean at the 47 ineffective sites $(177 \pm 161$ msec; $P=0.1$. The sensitivity of a PPI-VTCL difference $\leq 30 \mathrm{msec}$ for identifying an effective ablation site was $46 \%$, the specificity $64 \%$, the positive predictive value $41 \%$, and the negative predictive value $68 \%$.

Conclusion: The PPI-VTCL difference is not useful for discriminating between sites of concealed entrainment that are within or outside of a VT reentry circuit in patients with prior infarction. Therefore, in patients with prior infarction, the PPI is not clinically useful for identifying sites of concealed entrainment at which RF ablation should or should not be attempted. (J Cardiovasc Electrophysiol, Vol. 10, pp. 43-51, January 1999)
\end{abstract}

ventricular tachycardia, concealed entrainment, postpacing interval

\section{Introduction}

Concealed entrainment, also referred to as entrainment with concealed fusion, ${ }^{1,2}$ may be helpful in identifying a critical zone of slow conduction within a ventricular tachycardia (VT) reentry circuit in patients with prior myocardial infarction. ${ }^{1-5}$ However, the phenomenon of con-

Address for correspondence: Fred Morady, M.D., University of Michigan Medical Center, 1500 East Medical Center Dr., Box 0022, Ann Arbor, MI 48109-0022. Fax: 313-936-7026.

Manuscript received 5 July 1998; Accepted for publication 13 October 1998 cealed entrainment is not specific for a critical zone of slow conduction, and also may be demonstrated at bystander sites that are not essential for the maintenance of reentry. ${ }^{1-5}$ The postpacing interval (PPI) has been used to discriminate bystander sites from critical sites within the reentry circuit, with a PPI that is similar to the VT cycle length (CL) being indicative of a site within the reentry circuit. ${ }^{1}$

Although sound in theory and validated by a computer model of reentry, ${ }^{1}$ the clinical value of the PPI as a guide for ablation of VT has been critically evaluated in only a small number of studies. ${ }^{1,6}$ Therefore, the purpose of this study 
was to assess the extent to which the PPI helps to identify effective target sites for ablation of VT at sites of concealed entrainment in patients with prior myocardial infarction.

\section{Methods}

\section{Characteristics of Patients}

The subjects of this study were 24 patients with coronary artery disease and a past history of myocardial infarction who underwent an electrophysiology procedure for the purpose of radiofrequency (RF) ablation of hemodynamically tolerated VT. There were 19 men and 5 women, and their mean age was $66 \pm 12$ years $( \pm S D)$. The mean left ventricular ejection fraction was $0.27 \pm 0.09$. The site of prior myocardial infarction was anterior in 12 patients, inferior in 8 patients, and both anterior and inferior in 4 patients. The indications for catheter ablation were incessant VT in 4 patients, frequent implantable cardioverter defibrillator discharges in 15 patients, and recurrent drug-refractory VT associated with palpitations in 5 patients. Twenty-two of the 24 patients were being treated with an antiarrhythmic drug at the time of the catheter ablation procedure: amiodarone in 19 patients, sotalol in 2 patients, and quinidine in 1 patient. A total of 36 VTs were mapped and targeted for ablation. The mean VT CL was $483 \pm 80 \mathrm{msec}$. Eleven of the 24 patients in this study also were included in a prior study. ${ }^{6}$

\section{Electrophysiology Study}

The electrophysiology procedures were performed in the fasting state after informed consent was obtained. A quadripolar electrode catheter was positioned in the right ventricle for programmed ventricular stimulation, and a deflectable 7-French quadripolar electrode catheter with interelectrode spacing of 2-5-2 mm, a 4-mm distal electrode, and a thermistor in the distal electrode (EP Technologies, Mountainview, CA, USA) was inserted into the left ventricle for mapping and ablation. A retrograde aortic approach into the left ventricle was used in 23 patients, and a transseptal approach was used in 1 patient.

Pacing was performed at twice the diastolic threshold and with a pulse width of $2 \mathrm{msec}$ with a programmable stimulator (Bloom Associates
Ltd., Reading, PA, USA). The intracardiac electrograms and leads I, II, III, and $\mathrm{V}_{1}$ were recorded on paper at a paper speed of $100 \mathrm{~mm} / \mathrm{sec}$ (Mingograf-7, Siemens-Elema, Solna, Sweden) and also were stored on optical disk (EP Lab, Quinton, Bothell, WA, USA).

\section{Mapping of $V T$}

If incessant VT was not present, VT was induced by programmed ventricular stimulation with four extrastimuli. Depending on the site of prior infarction and the configuration of the VT, mapping was commenced either on the anterior or inferior wall of the left ventricle, or on the left ventricular septum. At sites where there was an abnormal electrogram, defined by an amplitude $\leq 0.5 \mathrm{mV}$ and a duration $\geq 60 \mathrm{msec}$, pacing trains of 10 to 15 stimuli were introduced at a CL 20 to $80 \mathrm{msec}$ (mean $46 \pm 20 \mathrm{msec}$ ) shorter than the VT CL. If necessary, the current strength was increased as high as $10 \mathrm{~mA}$, and the pulse width as high as $9 \mathrm{msec}$ to obtain capture.

Pacing in the left ventricle was performed in a bipolar configuration with electrodes 1 and 3 of the mapping/ablation catheter. Electrograms were recorded with electrodes 2 and 4 of the mapping/ablation catheter. This overlapping configuration of pacing and recording electrodes was used to minimize the disparity between the pacing and recording sites. The electrograms recorded in the left ventricle were filtered at 50 to $500 \mathrm{~Hz}$ and amplified to gain settings of 20 to 80 $\mathrm{mm} / \mathrm{mV}$.

\section{Ablation of VT}

The only criterion used in this study to select target sites for VT ablation was concealed entrainment. Concealed entrainment was defined as entrainment of VT at several pacing CLs 20 to $100 \mathrm{msec}$ shorter than the VT CL, with identical QRS complexes during pacing and during VT in each of the 12 electrocardiographic leads. The RF energy was delivered at a frequency of 500 $\mathrm{kHz}$ (EP Technologies).

The power output was adjusted automatically to maintain a preset electrode-tissue interface temperature of $60^{\circ} \mathrm{C}$. Applications of RF energy were delivered during VT, and were discontinued if a temperature of $\geq 55^{\circ} \mathrm{C}$ was not attained, or if the VT did not terminate during the first 20 seconds of an energy application. Applications of energy that were effective in terminating VT 
were continued for 60 seconds. Ablation was considered to be successful if the VT terminated during the energy application and was no longer inducible by programmed ventricular stimulation. Ineffective target sites were defined as sites at which RF energy did not terminate or prevent the reinduction of VT despite an electrode-tissue interface temperature of $\geq 55^{\circ} \mathrm{C} .{ }^{6}$

\section{Analysis of PPI}

PPIs did not influence the selection of target sites for ablation, and were measured only in a post hoc analysis. This study design allowed for determination of the sensitivity and specificity of the PPI for identification of critical sites within the reentry circuit.

The difference between the PPI and the VT CL (PPI-VTCL difference) was measured by determining the point at an interval after the last stimulus equal to the VT CL, then measuring the interval from this point to the first nonentrained electrogram (Fig. 1). Similar to Stevenson et al., ${ }^{1}$ if the point at an interval after the last stimulus equal to the VT CL fell within a fractionated electrogram, the PPI-VTCL difference was considered to be zero (Fig. 2). Because the stimulusQRS interval during concealed entrainment sometimes may be almost as long as the VT CL, it is possible for the point at an interval after the last stimulus equal to the VT CL to fall within the last entrained electrogram (Fig. 3). However, if a catheter is positioned within a critical zone of slow conduction, the interval between the stimulus and the last entrained electrogram during concealed entrainment is determined by the proximity of the pacing site to the exit site of the common pathway, ${ }^{7}$ and therefore does not reflect the time required for a complete revolution through the reentry circuit. Since the interval between the stimulus and the last entrained electrogram does not represent one revolution through the reentry circuit as intended by the PPI, the PPI in this situation would not be expected to discriminate between sites within and outside of the reentry circuit. Therefore, if the point at an interval after the last stimulus equal to the VT CL fell within the last entrained electrogram, the PPI-VTCL difference was considered to be the interval from that point to the first nonentrained electrogram (Fig. 3).

\section{Statistical Analysis}

Continuous variables are expressed as mean \pm SD and were compared using Student's $t$-test. Discrete variables were compared by contingency table analysis. $\mathrm{P}$ values $<0.05$ were considered significant.

\section{Results}

\section{Results of Ablation}

RF energy was delivered at 73 sites in the left ventricle where concealed entrainment was demonstrated during 36 VTs. At 47 of the 73 sites, an application of RF energy was ineffective, while at 26 of these sites, VT was successfully ablated. Ten of the VTs were not ablated by an energy application at a site where concealed entrainment was demonstrated.

\section{PPI-VTCL Difference}

The PPI-VTCL differences at the 26 effective ablation sites were compared with those at the 47 ineffective sites. The mean PPI-VTCL difference at the 26 effective sites was $114 \pm 137 \mathrm{msec}$, compared with a mean of $177 \pm 161 \mathrm{msec}$ at the 47 ineffective sites $(\mathrm{P}=0.1$ ) (Fig. 4).

\section{Sensitivity, Specificity, and Predictive Values}

A PPI-VTCL difference of $\leq 30 \mathrm{msec}$ has been considered to be useful in identifying effective target sites for terminating VT. ${ }^{1,8}$ Therefore, the results of this study were analyzed to determine the sensitivity, specificity, and positive and negative predictive values of a PPI-VTCL difference $\leq 30 \mathrm{msec}$ for identifying an effective ablation site. The sensitivity of a PPI-VTCL difference $\leq 30 \mathrm{msec}$ for identifying an effective ablation site was $46 \%$, the specificity $64 \%$, the positive predictive value $41 \%$, and the negative predictive value $68 \%$ (Table 1).

To determine if the sensitivity, specificity, and positive and negative predictive values of the PPI-VTCL difference for identifying an effective ablation site are influenced by the minimum acceptable PPI-VTCL difference, these values were also calculated for PPI-VTCL differences of $\leq 10, \leq 20, \leq 40$, and $\leq 50 \mathrm{msec}$ (Table 1). There were no significant differences between the sensitivities, specificities, or positive or neg- 

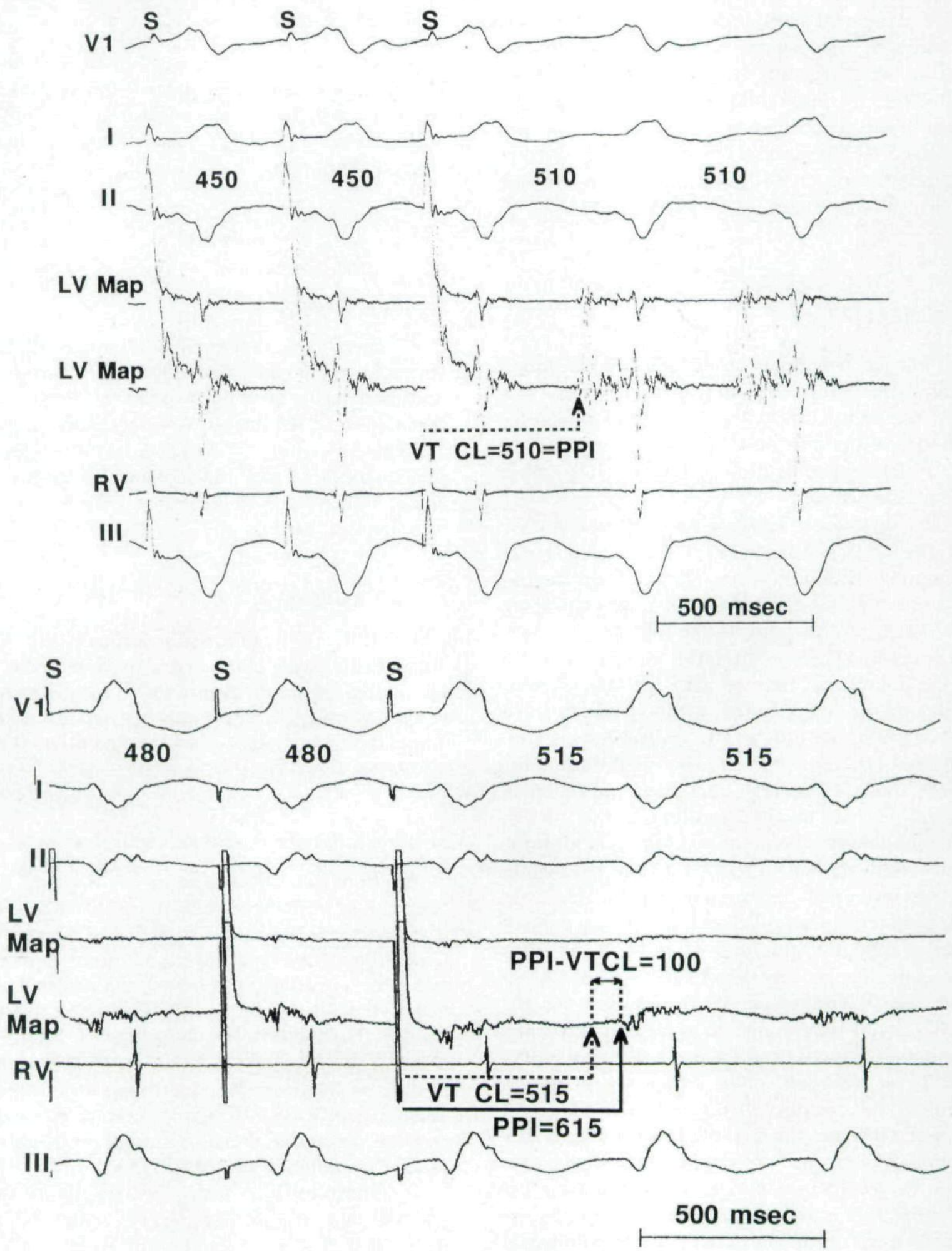

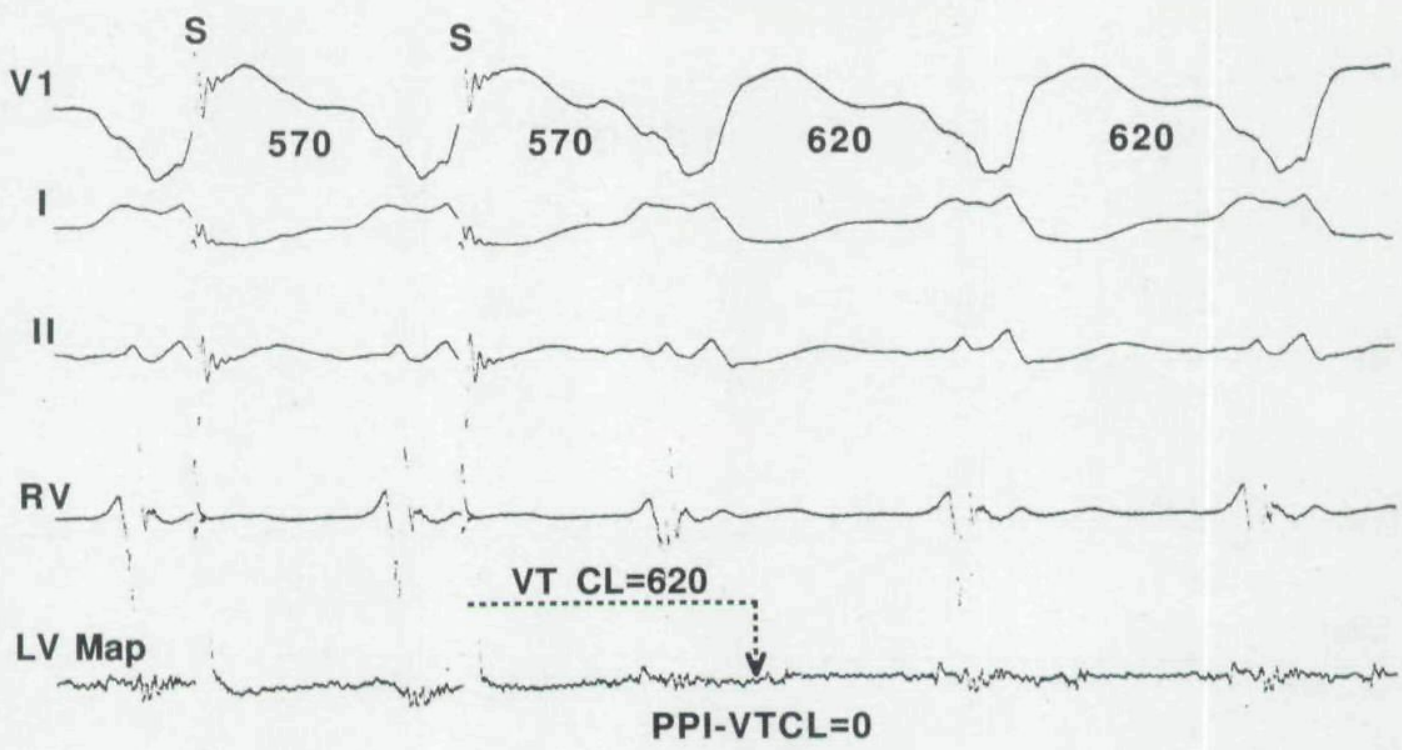

\section{Map}

III

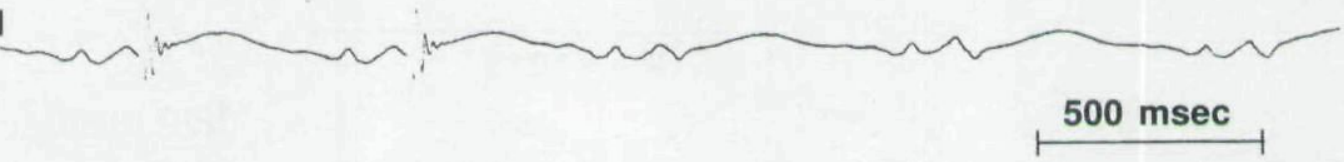

Figure 2. A postpacing interval (PPI) and ventricular tachycardia (VT) cycle length $(C L)(P P I-V T C L)$ difference of zero, recorded at an effective ablation site. The VT CL is $620 \mathrm{msec}$, and pacing at a CL of 570 msec with the mapping catheter resulted in concealed entrainment. The dashed arrow indicates the point after the last stimulus at an interval equal to the VT $C L(620 \mathrm{msec})$. Because this point falls within a broad, fractionated electrogram, the PPI-VTCL difference was considered to be zero. Abbreviations as in Figure 1.

ative predictive values of PPI-VTCL differences of $\leq 10, \leq 20, \leq 30, \leq 40$, or $\leq 50 \mathrm{msec}$.

\section{Discussion}

\section{Main Findings}

This study was designed to determine the extent to which the PPI is helpful in distinguishing whether a site of concealed entrainment is a bystander or active participant in the VT reentry circuit in patients with prior infarction. The results of this study demonstrate that the positive predictive value of a PPI-VTCL difference $\leq 30$ msec for identifying an effective ablation site is only $41 \%$, and that the negative predictive value is $68 \%$. In addition, the positive and negative predictive values of the PPI-VTCL difference are

Figure 1. Measurement of the difference between the postpacing interval (PPI) and the ventricular tachycardia (VT) cycle length (CL) (PPI-VTCL). (A) An example of a PPI-VTCL difference of zero, recorded at an ineffective ablation site. Shown are leads $V_{1}, I, I I$, and III, the intracardiac electrogram recorded by the left ventricular mapping catheter (LV map) at lowand high-gain settings, and an intracardiac electrogram recorded in the right ventricle (RV). The VT CL is $510 \mathrm{msec}$. Pacing with the mapping catheter at a CL of $450 \mathrm{msec}$ resulted in concealed entrainment, and the last three pacing stimuli (S) are shown. The point at an interval after the last stimulus equal to the VTCL $(510 \mathrm{msec})$ is indicated by the dashed arrow. Because this point coincides with the onset of the first nonentrained electrogram, the PPI-VTCL difference in this case is zero. (B) An example of a PPI-VTCL difference $>30$ msec at an effective ablation site. The VT CL is $515 \mathrm{msec}$, and pacing at a CL of 480 msec with the mapping catheter resulted in concealed entrainment. The dashed arrow indicates the point after the last stimulus equal to the VT CL $(515 \mathrm{msec})$. The PPI, measured from the last stimulus to the first nonentrained electrogram, is $615 \mathrm{msec}$, as indicated by the solid arrow. Therefore, in this case the PPI-VTCL difference was 100 msec. Of note is that the interval between the last stimulus and the last entrained electrogram is $115 \mathrm{msec}$, which is much shorter than the VTCL; use of this interval as the PPI would have yielded a theoretically unsound PPI-VTCL difference of $-400 \mathrm{msec}$. 

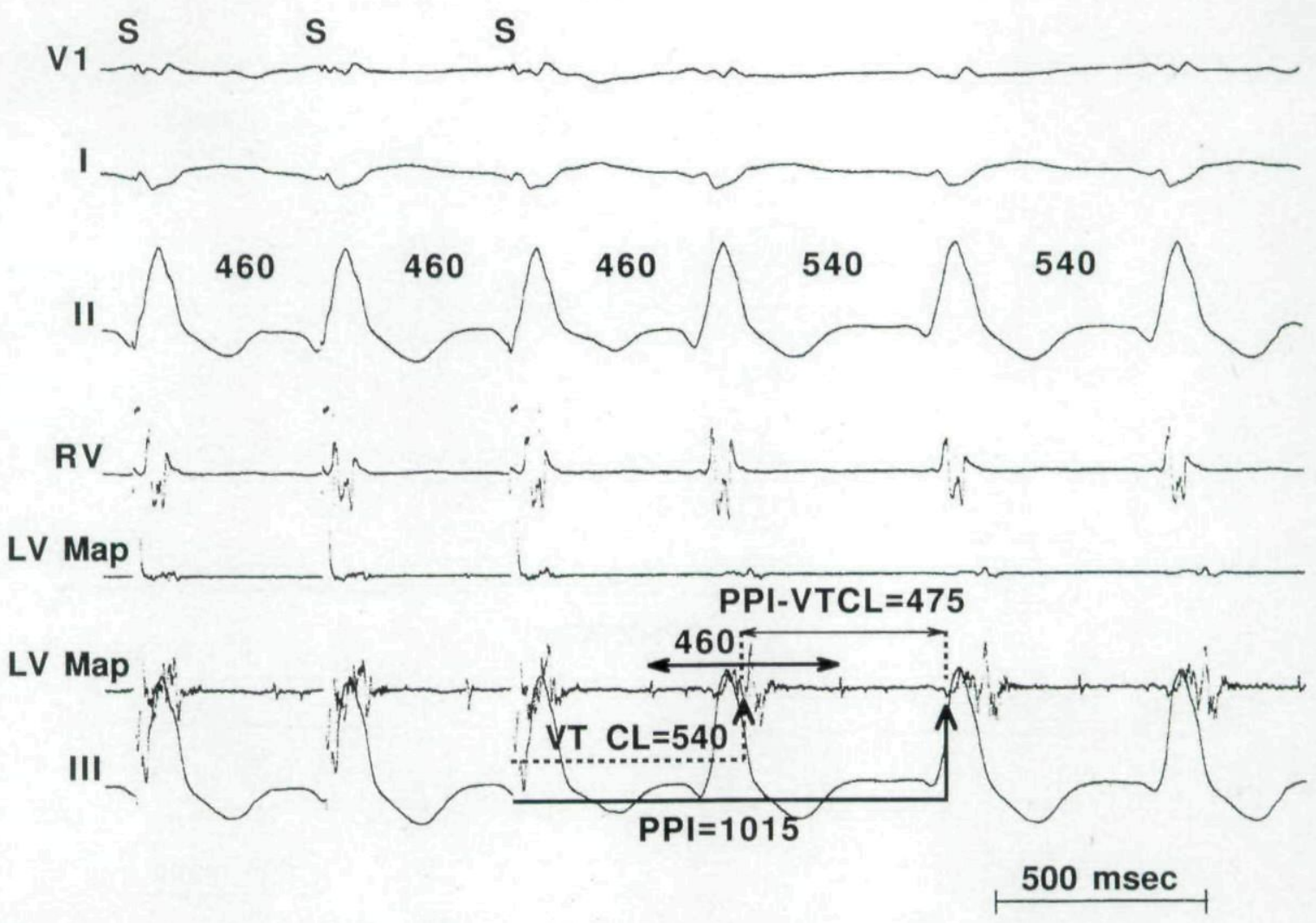

Figure 3. Measurement of the postpacing interval (PPI) and ventricular tachycardia (VT) cycle length (CL) (PPI-VTCL) difference at an unsuccessful ablation site where there was concealed entrainment with a long stimulus-QRS interval. The VT $C L$ is $540 \mathrm{msec}$, and pacing with the mapping catheter at a CL of 460 msec resulted in concealed entrainment, with a stimulus-QRS interval of $450 \mathrm{msec}$. The dashed arrow indicates the point after the last stimulus at an interval equal to the VT $C L(540 \mathrm{msec})$. This point falls within an electrogram, but this electrogram was not used for determining the PPI because it was entrained by the last pacing stimulus. The proof that this electrogram was entrained is that the interval between the two diastolic potentials on either side of the electrogram is $460 \mathrm{msec}$, equal to the pacing CL. Using the first nonentrained electrogram, the PPI interval is 1,015 msec, yielding a PPI-VTCL difference of $475 \mathrm{msec}$. Abbreviations as in Figure 1.

not significantly influenced by whether the minimum acceptable difference between the PPI and the VT CL is considered to be $10,20,30,40$, or $50 \mathrm{msec}$. Although the PPI theoretically should be reliable in differentiating bystander sites from sites within the reentry circuit, ${ }^{1}$ in this study, there was no significant difference between the mean PPI-VTCL differences at effective and ineffective ablation sites. Therefore, the results of this study indicate that the PPI-VTCL difference is not useful for discriminating between sites of concealed entrainment that are within or outside of the reentry circuit, at least for VT in the setting of prior infarction.

\section{Technique for Measuring the PPI}

If a stimulation site lies within a reentry circuit, the PPI is predicted to be equal to the VT
$\mathrm{CL}$, and if the site lies outside the reentry circuit, the PPI is predicted to be longer than the VT CL. ${ }^{1}$ In theory, unless the pacing alters the reentry circuit and changes the VT, there should be no situation in which pacing during VT results in a PPI that is shorter than the VT CL. ${ }^{1}$ Yet, a prior study reported PPIs as much as $100 \mathrm{msec}$ shorter than the VT CL. ${ }^{8}$ It is likely that this unexpected aberration in the PPI is a result of measurement technique. In the prior study, the PPI-VTCL difference was measured by determining the point at an interval equal to the VT CL after the last stimulus, and measuring the distance from this point "to the closest electrogram." 8 Whether or not the closest electrogram was the last entrained electrogram or the first nonentrained electrogram was not taken into consideration. However, when there is concealed entrainment, the interval between the last stimulus of a pacing train and the 


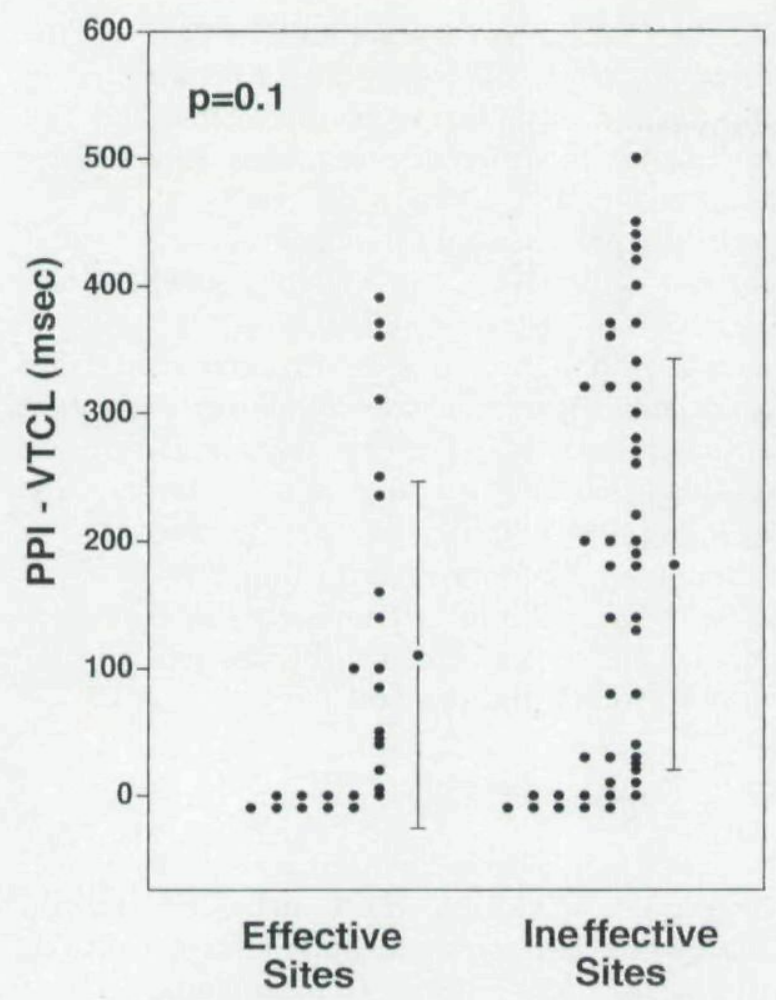

Figure 4. The individual postpacing interval and ventricular tachycardia cycle length (PPI-VTCL) differences measured at 26 effective ablation sites and 47 ineffective ablation sites. The mean $\pm S D$ s for each group also are shown.

last entrained electrogram is a function of the proximity of the pacing site to the exit site of the common pathway, ${ }^{7}$ and does not represent one revolution around the reentry circuit. Therefore, measurement of the PPI using the last entrained electrogram explains how a PPI may be found to be less than the VT CL (Fig. 1B). To avoid this problem, the PPI in the present study was measured to the first nonentrained electrogram.

\section{Limitations of the PPI}

A notable limitation of the PPI apparent from the results of this study is that this interval was more than $30 \mathrm{msec}$ longer than the VT CL at approximately $50 \%$ of sites that, because they were successful ablation sites, were very likely to be within the reentry circuit. The low sensitivity of a PPI-VTCL difference $\leq 30 \mathrm{msec}$ suggests that the measurement technique used in this study, namely the use of the first nonentrained electrogram for determination of the PPI, often yielded values that were not a valid indicator of the time for one revolution through the reentry circuit. Indeed, if the last entrained electrogram had been used for measuring the PPI, many of the PPI-VTCL differences found to be $>100$ msec would have been $\leq 30 \mathrm{msec}$, and the sensitivity of a PPI-VTCL difference $\leq 30 \mathrm{msec}$ for identifying a successful ablation site would have increased to $81 \%$. However, this also would have been the case at ineffective target sites (as in Fig. 3), such that there still would not have been a significant difference in the PPI-VTCL difference between effective and ineffective sites $(11 \pm 26$ and $15 \pm 51 \mathrm{msec}$, respectively; $\mathrm{P}=$ 0.7 ), and the specificity of a PPI-VTCL difference $\leq 30 \mathrm{msec}$ for identifying a successful ablation site would have dropped to only $17 \%$. Therefore, a fundamental problem with the PPI at sites of concealed entrainment in patients with prior myocardial infarction is that, regardless of the technique used for its measurement, it does not accurately indicate whether a site is within the reentry circuit or is a bystander site.

A characteristic of some effective ablation sites where the PPI-VTCL difference was $\geq 30 \mathrm{msec}$ was a long stimulus latency during concealed entrainment (e.g., a stimulus latency $>100 \mathrm{msec}$, as in Fig. 1B). For the PPI at a site within the reentry circuit to be a valid indicator of one revolution time in the circuit, the recorded electrogram must represent depolarization at or very close to the stimulation site. When the pacing site is within the reentry circuit and the stimulus latency is short, the interval between the stimulus and the first nonentrained electrogram reasonably can be expected to be equal to one revolution time in the circuit. ${ }^{1}$ However, if the interval between a stimulus and the electrogram that results from that stimulus is very long, as is sometimes the case when pacing in areas of slow

TABLE 1

Predictive Values of Postpacing Interval-Ventricular Tachycardia Cycle Length Difference for a Successful Ablation Site

\begin{tabular}{lcccc}
\hline $\begin{array}{l}\text { PPI- } \\
\text { VTCL }\end{array}$ & $\begin{array}{c}\text { Sensitivity } \\
\text { Difference }\end{array}$ & $\begin{array}{c}\text { Specificity } \\
(\boldsymbol{\%})\end{array}$ & $\begin{array}{c}\text { Positive } \\
\text { Predictive } \\
\text { Value } \\
(\boldsymbol{\%})\end{array}$ & $\begin{array}{c}\text { Negative } \\
\text { Predictive } \\
\text { Value } \\
(\%)\end{array}$ \\
\hline$\leq 10 \mathrm{msec}$ & 42 & 74 & 48 & 70 \\
$\leq 20 \mathrm{msec}$ & 46 & 72 & 48 & 71 \\
$\leq 30 \mathrm{msec}$ & 46 & 64 & 41 & 68 \\
$\leq 40 \mathrm{msec}$ & 50 & 62 & 42 & 69 \\
$\leq 50 \mathrm{msec}$ & 58 & 62 & 45 & 73 \\
\hline
\end{tabular}

These results were derived from 26 successful and 47 unsuccessful ablation sites. There were no significant differences in sensitivity, specificity, positive predictive value, or negative predictive value between the various PPI-VTCL differences. Abbreviations: PPI = postpacing interval; VTCL $=$ ventricular tachycardia cycle length . 
conduction (Fig. 1B), the interval from the stimulus to the first nonentrained electrogram will be longer than one revolution time, with the discrepancy being equal to the degree of stimulus latency. Therefore, a potentially effective target site at which there is concealed entrainment may be incorrectly judged to be a bystander site by relying on the PPI when there is a long stimulus latency.

The results of this study demonstrate a PPIVTCL difference $\leq 30 \mathrm{msec}$ at more than one third of ineffective ablation sites. This limitation in part may be related to the difficulty in determining the precise time of local activation when an electrogram is broad and fractionated, as is often the case at potential target sites in patients with prior infarction. Because of the inability to pinpoint the time of local activation when electrograms are broad and fractionated, the PPIVTCL difference, both in prior studies ${ }^{1,8}$ and in the present study, has been considered to be zero whenever the point at an interval after the last stimulus equal to the VT CL fell within a fractionated electrogram. Because this technique minimizes the difference between the PPI and the VT CL, it is likely to diminish the specificity of a PPI-VTCL difference of zero.

Another limitation of the PPI is that the zone of slow conduction of the reentry circuit often has decremental conduction properties. ${ }^{4}$ Therefore, even when the pacing site is within the reentry circuit, pacing at a CL shorter than that of the VT may result in a PPI that is longer than the VT CL. In prior studies, the pacing CL was as much as 100 msec shorter than the VT CL, ${ }^{1,6}$ which could have adversely affected the accuracy of the PPI. In the present study, pacing CLs were a mean of approximately $40 \mathrm{msec}$ shorter than the VT CL, and rate-related decremental conduction should have been minimal; nevertheless, rate-related conduction delay may have contributed to the inaccuracy of the PPI-VTCL difference as an indicator of proximity to the reentry circuit.

\section{Unipolar Versus Bipolar Pacing}

In some prior studies, unipolar pacing has been used for measurement of the PPI to avoid the possible inaccuracy introduced by anodal capture at the proximal pole. ${ }^{1.8}$ However, it often is not possible to record an electrogram with the pacing electrode upon cessation of unipolar pacing. Therefore, unipolar pacing with the distal electrode of a mapping catheter often necessitates the use of electrodes 3 and 4 to record a bipolar electrogram several millimeters from the distal electrode. Even so, the PPI measured in this fashion usually is very similar to the PPI obtained with a bipolar electrogram recorded by electrodes 1 and 2.8

In the present study, bipolar pacing was performed with electrodes 1 and 3 , and bipolar electrograms were recorded with electrodes 2 and 4. Although bipolar pacing may have been associated with anodal capture, this configuration allowed for overlap between the stimulation site and the recording site, and this would not have been possible with unipolar pacing. Because the stimulation bipole and recording bipoles were offset by only $2 \mathrm{~mm}$, it is unlikely that the use of bipolar pacing accounts for the shortcomings of the PPI found in this study.

\section{Prior Studies}

The positive predictive value of a PPI-VTCL difference $\leq 30 \mathrm{msec}$ for a successful ablation site of $41 \%$ in the present study is higher than or similar to values reported in prior studies. ${ }^{1.6}$ In a prior study of patients with VT and prior infarction, the positive predictive value of a PPI-VTCL difference $\leq 30 \mathrm{msec}$ at sites of concealed entrainment was only $26 \% .^{1}$ A likely explanation for this discrepancy is the different techniques used to measure the PPI in the two studies. As explained above, in the present but not in the prior study, ${ }^{1}$ the PPI was measured to the first nonentrained electrogram. Had the next electrogram after the last stimulus been used regardless of whether or not it was entrained, the positive predictive value of a PPI-VTCL difference $\leq 30$ msec in the present study would have dropped from $41 \%$ to $35 \%$, closer to the $26 \%$ positive predictive value reported in the prior study. ${ }^{1}$

Another possible explanation for the lower positive predictive value of a PPI-VTCL difference $\leq$ $30 \mathrm{msec}$ in the prior study is that RF energy was delivered at power settings of 20 to $35 \mathrm{~W}$, without temperature monitoring. Applications of 20 to 35 $\mathrm{W}$ may be insufficient for adequate tissue heating, particularly in areas of scar.9.10 Inadequate tissue heating at sites that actually were within the reentry circuit would lead to underestimation of the positive predictive value of a PPI-VTCL difference $\leq$ $30 \mathrm{msec}$. In the present study, applications of RF energy were guided by temperature monitoring, and a site was not considered to be an ineffective ablation site unless an electrode-tissue interface temperature of $55^{\circ} \mathrm{C}$ was achieved. In a prior study 
in which RF applications also were guided by temperature monitoring, the positive predictive value of a PPI-VTCL difference $\leq 30 \mathrm{msec}$ was found to be $45 \%$, similar to the results of the present study. ${ }^{6}$

\section{Limitations}

A limitation of this study is that the response to RF ablation was the only criterion available for judging whether or not a pacing site was within a critical portion of the reentry circuit. Because the area that was ablated may not have coincided exactly with the area that was paced, this criterion may have been imprecise. In addition, it is possible that some pacing sites were within the reentry circuit, at sites too large to be interrupted by a single application of radiofrequency energy; such pacing sites would have been incorrectly classified as lying outside the reentry circuit.

All of the patients in this study had coronary artery disease and a history of myocardial infarction. Because the electrogram characteristics and conduction properties of other substrates are likely to be different, the results of this study cannot be applied to use of the PPI for atrial arrhythmias or for VT in patients without coronary artery disease.

\section{Conclusion}

In conclusion, although the PPI theoretically should be helpful in differentiating critical sites within a reentry circuit from bystander sites, it has no discriminative value at sites of concealed entrainment in patients with prior infarction. Probably because of limitations inherent in recording and interpreting electrograms at sites of abnormal conduction, the PPIs at sites of concealed entrainment that are within a critical zone of slow conduction often do not accurately reflect one revolution time around the reentry circuit. Furthermore, regardless of the technique used to measure the PPI, the mean PPI-VTCL differences at effective and ineffective ablation sites do not differ significantly. Therefore, the PPI is not clinically useful for identifying sites of concealed entrainment at which RF ablation should or should not be attempted. Other criteria, including isolated diastolic potentials that cannot be dissociated from the reentry circuit, a stimulus-QRS/VT CL ratio $<0.7$, and a stimulus-QRS interval equal to the electrogram-QRS interval, may be more helpful in selecting sites of con- cealed entrainment at which an attempt at VT ablation is appropriate. ${ }^{6}$ However, although their clinical value as a guide for ablation is greater than that of the PPI, none of these criteria are $100 \%$ sensitive or specific for successful ablation at sites of concealed entrainment. ${ }^{1,6}$ Therefore, it may be clinically appropriate to deliver a test application of energy during VT at all sites at which concealed entrainment is demonstrated.

Acknowledgment: The authors thank Dr. Alan Kadish for his critical review of this manuscript.

\section{References}

1. Stevenson WG, Khan H, Sager P, et al: Identification of reentry circuit sites during catheter mapping and radiofrequency ablation of ventricular tachycardia late after myocardial infarction. Circulation 1993;88:1647-1670.

2. Stevenson WG, Sager P, Friedman PL: Entrainment techniques for mapping atrial and ventricular tachycardias. J Cardiovasc Electrophysiol 1995;6:201-216.

3. Stevenson WG, Weiss J, Wiener I, et al: Localization of slow conduction in a ventricular tachycardia circuit: Implications for catheter ablation. Am Heart J 1987; 114:1253-1258.

4. Morady F, Frank R, Kou WH, et al: Identification and catheter ablation of a zone of slow conduction in the reentrant circuit of ventricular tachycardia in humans. J Am Coll Cardiol 1988;11:775-782.

5. Morady F, Kadish A, Rosenheck S, et al: Concealed entrainment as a guide for catheter ablation of ventricular tachycardia in patients with prior myocardial infarction. J Am Coll Cardiol 1991;17:678-689.

6. Bogun F, Bahu M, Knight BP, et al: Comparison of effective and ineffective target sites that demonstrate concealed entrainment in patients with coronary artery disease undergoing radiofrequency ablation of ventricular tachycardia. Circulation 1997;95:183-190.

7. Bogun F, Bahu M, Knight BP, et al: Response to pacing at sites of isolated diastolic potentials during ventricular tachycardia in patients with previous myocardial infarction. J Am Coll Cardiol 1997;30:505-513.

8. Hadjis TA, Harada T, Stevenson WG, et al: Effect of recording site on postpacing interval measurement during catheter mapping and entrainment of postinfarction ventricular tachycardia. J Cardiovasc Electrophysiol 1997;8:398-404.

9. Bartlett TG, Mitchell R, Friedman PL, et al: Histologic evolution of radiofrequency lesions in an old human myocardial infarction causing ventricular tachycardia. J Cardiovasc Electrophysiol 1995;6:625-629.

10. Kottkamp H, Hindricks G, Horst E, et al: Subendocardial and intramural temperature response during radiofrequency catheter ablation in chronic myocardial infarction and normal myocardium. Circulation 1997;95: 2155-2161. 
This document is a scanned copy of a printed document. No warranty is given about the accuracy of the copy. Users should refer to the original published version of the material. 